\title{
The Phase Transition for Zinc Sulfide Nanosheets under High Pressure
}

Zepeng $\mathrm{Li}^{1,2}$, Jinhua Wang ${ }^{2,3}$, Bingbing $\mathrm{Liu}^{2} *$, Jing Liu ${ }^{4}$

${ }^{1}$ School of Science, Civil Aviation University of China, Tianjin 300300, China.

${ }^{2}$ State Key Laboratory of Superhard Materials, Jilin University, Changchun 130012, China.

${ }^{3}$ School of Science, Tianjin University of Technology and Education, Tianjin 300222, China

${ }^{4}$ Institute of High Energy physics, Chinese Academy of Sciences, Beijing 100023, China

* Corresponding Author

E-mail: 1i_zepeng@163.com, liubb@jlu.edu.cn 


\section{Support information}

The additional author names after ten for ref.13, 21, 27 and 29:

(13) Hemley, R. J.; Mao, W. L.; Mao, H. K.

(21) Cui, T.; Zou, G. T.; Wang, G. R.; Liu, Y. C.

(27) Kondoe, T.; Yagie, T.

(29) Hahn, H.; Gleiter, H.; Jiang, J. Z. 